\title{
Réponse des communautés d'oligochètes (Tubificidés et Lumbriculidés) et de diptères Chironomidés à la baisse des teneurs en phosphore dans le Léman (Petit Lac)
}

\author{
C. Lang 1
}

Mots-clés : Chironomidés, Oligochaeta, zoobenthos, indicateur, eutrophisation, lac, restauration.

Une campagne de prélèvements réalisée en 1999, de façon comparable à celle effectuée en 1994, montre que la composition des communautés d'oligochètes et de Chironomidés a changé dans cet intervalle de temps en réponse à la baisse des concentrations en phosphore dans l'eau du Petit Lac, le bassin ouest du Léman (Suisse et France). Les larves de Chironomidés étaient présentes dans $66 \%$ des 159 sites visités en 1999 contre $40 \%$ en 1994 tandis que l'abondance des oligochètes a diminué. De plus, l'abondance relative des taxons indicateurs de conditions oligotrophes (Micropsectra et Spirosperma velutinus) a augmenté en 1999. Ces changements indiquent qu'en 1999 la restauration biologique des sédiments profonds se trouve à mi-chemin entre l'état méso-eutrophe des environs de 1980 et l'état oligotrophe d'avant 1950. Cependant, l'ampleur de la restauration n'est pas la même dans les trois bassins du Petit Lac: elle varie en fonction de l'intensité de la sédimentation organique.

Response of oligochaete (Tubificidae and Lumbriculidae) and Diptera Chironomidae communities to the decrease of phosphorus concentrations in Lake Geneva (Little Lake)

Keywords : chironomid, oligochaete, zoobenthos, indicator, eutrophication, lake, recovery.

A survey carried out in 1999, in the same way as in 1994, indicated that the composition of oligochaete and chironomid communities changed during this period in response to the decrease of phosphorus concentrations in the water of the western basin (the Little Lake) of Lake Geneva (Switzerland and France). Chironomid larvae were present in $66 \%$ of 159 sitess in 1999 agaainst $40 \%$ in 1994 whereas the abundance of oligochaetes decreased. In addition, the relative abundance of taxa indicator of oligotrophic conditions (Micropsectra and Spirosperma velutinus) was greater in 1999. According to these changes, the biological recovery of deep sediment was in 1999 mid-way between the meso-eutrophic state prevailing around 1980 and the oligotrophic state prevailing before 1950 . However, the extent of recovery was not the same in the three basins of the Little Lake: it varied according to the intensity of organic deposition.

\section{Introduction}

En Europe occidentale, les apports en phosphore dans les lacs tendent à diminuer grâce à la généralisation des stations d'épuration capables de fixer cet élément, à la réduction des concentrations dans les lessives et à un meilleur contrôle des pratiques agricoles (Sas 1989). Toutefois, la baisse des concentrations en

1. Conservation de la faune, Marquisat 1, CH-1025 St-Sulpice, Suisse.

E- mail: claude.lang @ sffn.vd.ch phosphore dans l'eau des lacs n'entraîne pas nécessairement la diminution espérée des algues planctoniques. Celles-ci peuvent persister en abondance et, par l'intermédiaire de la sédimentation, affecter les qualités chimiques et biologiques des sédiments (Baines \& Pace 1994). C'est la restauration de ces qualités qui montrera que l'état d'un lac s'est vraiment amélioré puisque tout ce qui est produit dans la colonne d'eau finit par aboutir, sous une forme ou une autre, sur le sédiment (Hakanson \& Jansson 1983). Dans cette optique, l'analyse de la composition des communautés du zoobenthos (Oligochètes et Chironomidés surtout) constitue un des moyens les plus simples d'éva- 
luer l'amélioration de l'état des sédiments (Johnson et al. 1993). C'est l'approche choisie dans cette étude pour suivre les progrès de la restauration biologique du Petit Lac, le bassin ouest du Léman, et les comparer à ceux du reste du lac (Lang 1998).

En effet, en réponse aux mesures d'assainissement prises, les concentrations en phosphore total diminuent régulièrement dans l'eau du Léman : passant d'un maximum de près de $90 \mathrm{mg} / \mathrm{m}^{3}$ en 1979 à moins de $40 \mathrm{mg}$ en 1998 et se rapprochant ainsi des $12 \mathrm{mg}$ mesurés en 1957 (Blanc et al. 1999). Le phytoplancton ne répond pas encore de façon claire à la baisse du phosphore : certaines années c'est la biomasse qui baisse, d'autres années c'est la production primaire qui diminue sans qu'il soit possible de discerner des tendances à long terme (Druart et al. 1999, Pelletier \& Leboulanger 1999). Au contraire, l'évolution des communautés de macrophytes de la zone littorale, avec le retour en force des characées (Demierre \& Durand 1999), montre qu'en 1998 le Léman se rapproche lentement de l'état oligotrophe qui était le sien, probablement jusqu'à la fin des années quarante.

Les Oligochètes et les Chironomidés présents entre 1954 et 1966 dans la zone profonde, lorsque l'état du Léman a commencé à se dégrader, sont bien connus (Juget 1967). C'est à ce moment que les espèces indicatrices de conditions oligotrophes ont amorcé une diminution qui s'est poursuivie jusqu'en 1982 sous l'effet de l'augmentation du phosphore (Lang 1998, LodsCrozet \& Lachavanne 1994). Au contraire, lorsque la concentration de ce nutriment a diminué, le pourcentage moyen d'individus appartenant à ces espèces indicatrices a augmenté : passant de $17 \%$ en 1982 à $41 \%$ en 1991 dans les communautés d'oligochètes (Lang 1998) et se rapprochant ainsi de $70 \%$, la valeur moyenne qui caractérise les lacs oligotrophes, tels que le Lac Supérieur en Amérique du Nord et le Léman au début du siècle (Lang 1990).

Toutefois, l'amélioration indiquée par le zoobenthos en 1991 ne se produit pas à la même vitesse dans l'ensemble du Léman (Lang 1998). Dans le Grand Lac (le bassin principal du Léman : $503 \mathrm{~km}^{2}$ sur un total de $582 \mathrm{~km}^{2}$ ), elle varie en fonction de la profondeur : plus lente à -150 m qu'à $-40 \mathrm{~m}$, elle n'a pas encore commencé dans la zone la plus profonde $(-290 \mathrm{~m}$ à $-309 \mathrm{~m})$. Les espèces d'oligochètes indicatrices de conditions oligotrophes, présentes en 1963 (Juget 1967), n'y sont pas revenues en 1998 (Lang 1999 b) à cause d'un manque d'oxygène persistant (Blanc et al. 1999). Dans d'autres zones, comme le Petit Lac (bassin ouest du Léman), où les concentrations en oxygène ne descendent jamais en dessous de $8 \mathrm{mg} / \mathrm{l}$ dans la zone profonde
(Blanc et al. 1996), c'est un excès de sédimentation organique qui en 1994 retarde la restauration biologique des sédiments (Lang \& Reymond 1996 a). Dans la présente étude, les communautés d'oligochètes et de Chironomidés, étudiées en 1999 dans le Petit Lac, sont comparées aux communautés de 1994 afin d'évaluer la réponse biologique des sédiments profonds à la baisse des concentrations en phosphore dans la colonne d'eau.

\section{Stations et méthodes}

Le Petit Lac qui est beaucoup moins profond que le Grand Lac (p. max. : 78 et 309 m ; p. moy. : 41 et $172 \mathrm{~m}$ ), est divisé en trois bassins : Chevrens, Tougues et Nyon (Fig.1) dont la profondeur maximale atteint au moins $70 \mathrm{~m}$. Ces bassins sont séparés les uns des autres ainsi que du Grand Lac par des seuils dont les profondeurs varient entre $55 \mathrm{~m}$ et $60 \mathrm{~m}$. Du fait de cette structure particulière et de la direction des vents et des courants dominants dans l'axe du lac (Lemmin et al. 1989), la sédimentation organique décroît entre le bas-

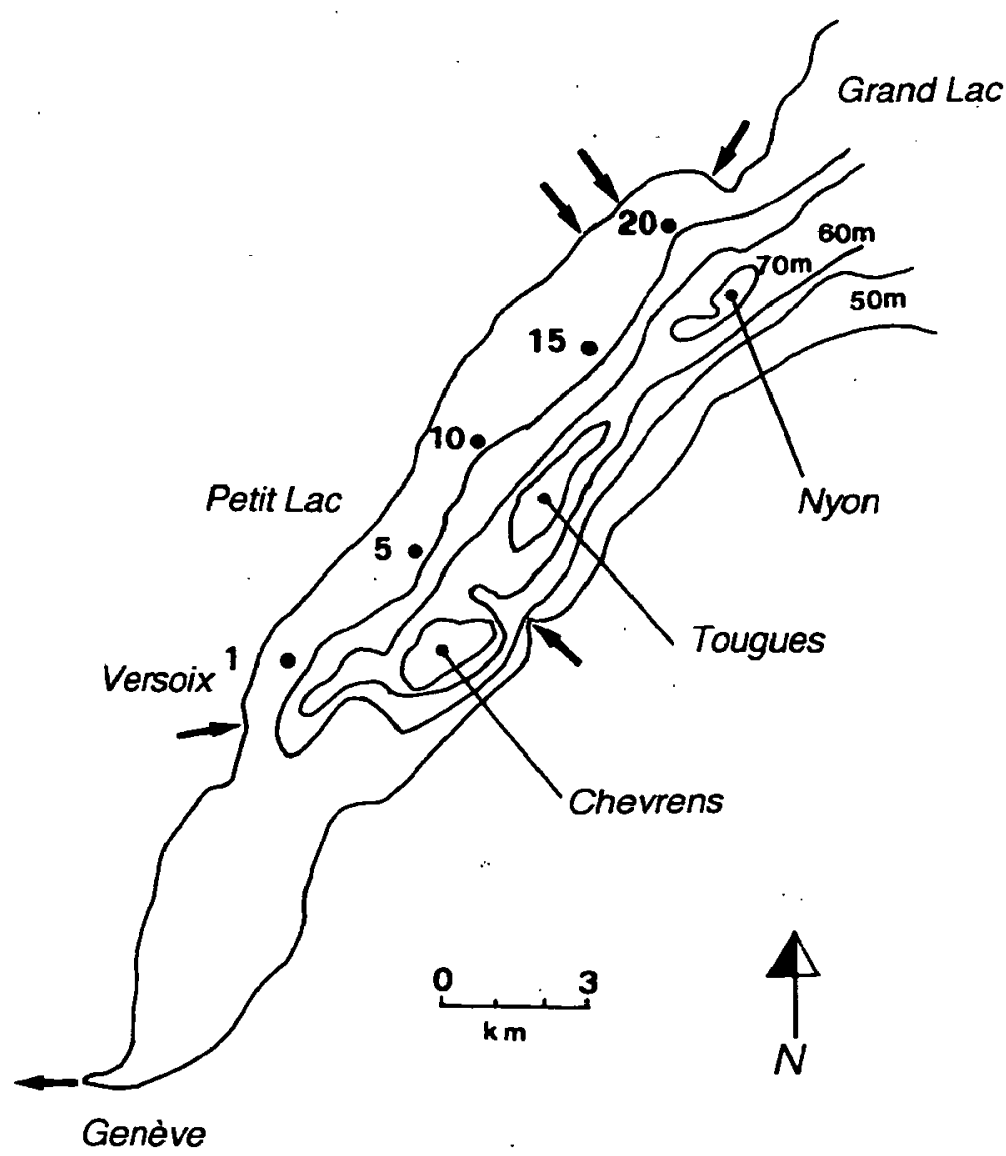

Fig. 1. Localisation des trois bassins et des 22 transects visités en 1994 et en 1999 dans le Petit Lac (Léman). Les points de départ de cinq transects sont indiqués par des cercles et des chiffres (1, $5,10,15,20)$. Les flèches représentent les principaux apports organiques par les rivières.

Fig. 1. Location of the three basins and of the 22 transects sampled in 1994 and in 1999 in the Little Lake (Lake Geneva). Circles and digits $(1,5,10,15,20)$ indicated the starting points of five selected transects. Arrows showed the main organic inputs by rivers. 
sin de Chevrens et celui de Nyon (Lang \& Reymond 1996 a).

Le zoobenthos présent dans la zone profonde (34-78 m) du Petit Lac a été étudié à deux reprises de façon absolument comparable : la première fois entre le 30 mai et le 8 juin 1994, la seconde fois entre le 19 mai et le $1^{\text {er juin } 1999}$ à partir d'un réseau de 159 stations de prélèvements, localisées au moyen d'un récepteur GPS. Ces stations, distantes de $500 \mathrm{~m}$ les unes des autres, sont placées sur 22 transects parallèles les uns aux autres. Le transect 1 suit la coordonnée 127 de la carte nationale de la Suisse, le transect 22 la coordonnée 137.5. Comme les transects sont également distants de $500 \mathrm{~m}$, l'ensemble de la zone profonde est ainsi couverte par un quadrillage régulier, correspondant à celui de la carte nationale de la Suisse. Les coordonnées géographiques des stations sont indiquées ailleurs (Lang \& Reymond 1996 b). Dans chaque station, une carotte de sédiment d'une longueur de $30 \mathrm{~cm}$, couvrant une surface de $16 \mathrm{~cm}^{2}$, est prélevée au moyen d'un carottier descendu depuis la surface.

Au laboratoire, l'épaisseur des trois couches verticales du sédiment récolté est mesurée avec, de la surface vers le fond, la couche brune oxydée, la couche noire réduite et la couche d'argile grise. L'épaisseur de la couche noire donne une estimation grossière de la quantité de matière organique déposée (Hakanson \& Jansson 1983) ce qui permet de classer les stations de prélèvements en fonction de l'intensité de la sédimentation organique (Lang 1998). Après cette inspection visuelle, le sédiment est tamisé (vide de maille : $0.2 \mathrm{~mm}$ ) et le refus du tamis est conservé dans du formol $5 \%$. Les tubifícidés, les lumbriculidés et les larves de Chironomidés récoltés sont ensuite séparés du sédiment et comptés sous une loupe. Seuls les oligochètes de diamètre $>0.29 \mathrm{~mm}$, sont montés et identifiés (Reymond 1994) afin d'éliminer les jeunes individus dont les variations saisonnières influencent trop l'estimation de l'abondance des espèces (Lang 1999a).

Les larves de Chironomidés sont identifiées d'après Wierderholm (1983) et la valeur indicatrice des taxons est reprise de Saether (1979). En 1999, les Chironomidés sont comptés et identifiés dans chaque carotte séparément ; en 1994 au contraire, si les larves sont également comptées carotte par carotte, elles sont seulement identifiées en bloc dans l'ensemble des 159 carottes.

Les espèces d'oligochètes sont classées en trois groupes selon leur valeur indicatrice (Lang 1990) : espèces des conditions oligotrophes (Tableau 1 : espèces 1 à 4), mésotrophes (espèces 5 à 7) ou eutrophes (Tableau 1 : variables 8 et 9 qui regroupent plusieurs espèces). L'espèce Potamothrix moldaviensis, bien qu'indicatrice de conditions mésotrophes, est classée dans la variable 8 avec deux espèces eutrophes du genre Limnodrilus parce que les immatures de ces trois espèces sont difficiles à différencier. Pour la même raison, les trois espèces eutrophes formant la variable 9 sont analysées en bloc. Pour chaque espèce ou pour chaque groupe d'espèces, la fréquence (le nombre de carottes dans lesquelles l'espèce est présente) et l'abondance (le nombre d'individus présents par carotte) sont déterminées. L'abondance relative des espèces indicatrices de conditions oligotrophes, appelées pour simplifier espèces oligotrophes (EO), est calculée en rapportant, sous forme de pourcentage, le nombre d'individus appartenant à ces espèces au nombre total deTubificidés et de Lumbriculidés adultes (diamètre $>0.29$ $\mathrm{mm})$ présents dans une carotte. En 1999, l'abondance relative des espèces d'oligochètes et de Chironomidés indicatrices de conditions oligotrophes est calculée pour chaque carotte de la même façon que pour les oligochètes ; en 1994, elle est calculée seulement à partir du nombre total d'individus présents dans 159 carottes parce que les Chironomidés n'ont pas été identifiés carotte par carotte.

Les variations de l'abondance des espèces entre 1994 et 1999 sont comparées à trois niveaux : les 159 stations, les 22 transects et les 3 bassins. Les abondances relatives moyennes de EO obtenues pour ces différents ensembles sont comparées aux valeurs de référence suivantes (Lang 1990) : EO est égal à zéro si les conditions à la surface du sédiment sont celles d'un milieu eutrophe, les valeurs de EO varient entre 1 et $17 \%$ dans un milieu méso-eutrophe, entre 18 et $52 \%$ (valeur médiane : $35 \%$ ) dans un milieu mésotrophe, entre 52 et $69 \%$ dans un milieu oligo-mésotrophe, enfin EO dépasse $69 \%$ dans un milieu oligotrophe.

L'abondance relative (\%) moyenne de EO peut également être calculée (EOC) à partir des concentrations moyennes $\left(\mathrm{mg} / \mathrm{m}^{3}\right)$ en phosphore total (PT) mesurées dans l'eau du lac au cours des 5 années précédant le prélèvement du zoobenthos (Lang 1990) :

$$
\text { EOC }=80.29-8.35 \text { PT }^{0.5} \quad\left(r^{2}=0.81, n=15\right) .
$$

Cette relation empirique est basée sur 15 campagnes effectuées dans 6 lacs d'Europe et 3 lacs d'Amérique $\mathrm{du}$ Nord dont le Lac Supérieur. Les concentrations moyennes du PT sur 5 ans sont utilisées parce que le zoobenthos ne réagit pas immédiatement à une variation de ce nutriment (Lang 1998). Si la valeur moyenne de EO observée dans une série de prélèvements est inférieure à EOC, la valeur calculée, cela signifie que la restauration de l'état biologique du sédiment est en retard par rapport à l'état trophique indiqué par PT. Ce 
Tableau 1. Fréquence et abondance relatives (\%) des taxons d'oligochètes et de Chironomidés identifiés dans le Petit Lac en 1994 et 1999 . Le tiret indique des résultats manquants.

Table 1. Relative frequency and abundance (\%) of oligochaete and chironomid taxa identified in the Little Lake in 1994 and 1999. The dash indicates missing data.

\begin{tabular}{|c|c|c|c|c|c|c|}
\hline \multirow[t]{2}{*}{ Variables } & \multirow[t]{2}{*}{ Taxons } & \multicolumn{3}{|c|}{ Fréquence ${ }^{1}$} & \multicolumn{2}{|c|}{ Abondance ${ }^{2}$} \\
\hline & & 1994 & 1999 & $\mathrm{P}\left(\mathrm{Chi}^{2}\right)$ & 1994 & 1999 \\
\hline 1 & Bichaeta sanguinea Bretscher & 16.4 & 19.5 & 0.465 & 2.8 & 3.6 \\
\hline 2 & Stylodrilus heringianus Claparède & 32.7 & 28.3 & 0.393 & 6.6 & 6.3 \\
\hline 3 & Stylodrilus lemani (Grube) & 5.7 & 4.4 & 0.608 & 0.9 & 0.6 \\
\hline 4 & Spirosperma velutinus (Grube) & 14.5 & 20.8 & 0.141 & 2.1 & 3.8 \\
\hline 5 & Potamothrix vejdovskyi (Hrabe) & 88.7 & 72.3 & 0.000 & 38.6 & 22.9 \\
\hline 6 & Psammoryctides barbatus (Grube) & 11.9 & 9.4 & 0.468 & 1.7 & 1.5 \\
\hline 7 & Spirosperma ferox (Eisen) & 4.4 & 8.8 & 0.114 & 0.6 & 1.3 \\
\hline 8 & $\begin{array}{l}\text { Limnodrilus hoffmeisteri (Claparède) } \\
\text { Limnodrilus profundicola Verrill } \\
\text { Potamothrix moldaviensis (Vejdovsky, } \\
\text { Mrazek) }\end{array}$ & 20.8 & 35.8 & 0.002 & 3.2 & 6.7 \\
\hline 9 & $\begin{array}{l}\text { Potamothrix heuscheri (Bretscher) } \\
\text { Potamothrix hammoniensis (Michaelsen) } \\
\text { Tubifex tubifex (Müller) }\end{array}$ & 83.0 & 83.0 & 1.000 & 35.7 & 36.4 \\
\hline 10 & Micropsectra & - & 37.7 & - & 2.1 & 10.0 \\
\hline 11 & Procladius & - & 30.2 & - & 4.6 & 5.6 \\
\hline 12 & Tanytarsus & - & 1.9 & - & 0.6 & 0.2 \\
\hline 13 & Autres Chironomidés ${ }^{3}$ & - & 0.1 & - & 0.4 & 1.1 \\
\hline 14 & Tous les Chironomidés & 39.6 & 66.0 & 0.000 & 7.7 & 16.9 \\
\hline 15 & Oligochètes oligotrophes, taxons $1-4$ & 52.2 & 51.6 & 0.911 & 12.4 & 14.4 \\
\hline 16 & Taxons oligotrophes $1-4$ et 10 & - & 67.3 & - & 14.5 & 24.3 \\
\hline
\end{tabular}

1. Pourcentage des 159 prélèvements dans lesquels un taxon est présent.

2. Pourcentage des 1328 (en 1994) et des 1351 (en 1999) individus identifiés dans 159 prélèvements appartenant à un taxon donné.

3. Dicrotendipes, Paracladopelma, Phaenopsectra.

retard peut être causé par un manque d'oxygène ou par un excès de sédimentation organique (Lang \& Reymond 1996 a).

\section{Résultats}

La comparaison globale (Tableau 1) des 159 stations montre qu'en 1999 la fréquence et l'abondance relatives de Potamothrix vejdovskyi diminuent par rapport à 1994 tandis que celles des deux espèces de Limnodrilus (associées à Potamothrix moldaviensis) et des larves de Chironomidés dont Micropsectra augmentent. L'abondance relative des espèces d'oligochètes et de Chironomidés indicatrices de conditions oligotrophes, calculée globalement, passe de $14.5 \%$ en 1994 à $24.3 \%$ en 1999 (test de $\mathrm{Chi}^{2}, \mathrm{P}=0.000$ ), l'augmentation étant surtout due à Micropsectra. Dans le même temps, les oligochètes restent présents dans tous les prélèvements mais leur abondance moyenne diminue, passant de 20237 ind. $/ \mathrm{m}^{2}$ en 1994 à 12450 en 1999 (test de $t$, stations appariées, $P=0.000$ ).
La comparaison entre les trois bassins du Petit Lac (Tableau 2) montre que, d'une manière générale, la fréquence des taxons indicateurs de conditions oligotrophes augmente entre le bassin de Chevrens et celui de Nyon (Fig. 1). Dans le bassin de Chevrens, la fréquence des taxons ne change pas significativement entre 1994 et 1999. Dans les bassins de Tougues et de Nyon, la fréquence des larves de Chironomidés augmente fortement en 1999. Notons également l'augmentation dans le bassin de Tougues de Spirosperma velutinus, un des meilleurs indicateurs de conditions oligotrophes dans le Léman (Lang 1998). Remarquons, à ce propos, que cette espèce est également devenue plus fréquente dans le bassin de Chevrens en 1999. C'est dans le bassin de Nyon que les changements sont les plus importants. Ils correspondent dans les grandes lignes à ceux déjà décrits dans la comparaison globale.

Les résultats présentés ci-dessus confirment le fait que la sédimentation organique décroît entre les bassins de Chevrens et de Nyon (Lang \& Reymond 1996 a). 
Tableau 2. Fréquence relative (\%) des taxons d'oligochètes et de Chironomidés identifiés en 1994 et en 1999 dans chacun des trois bassins du Petit Lac (voir Fig. 1).

Table 2. Relative frequency (\%) of oligochaete and chironomid taxa identified in 1994 and in 1999 in each of the three basins of the Little Lake (see Fig. 1).

\begin{tabular}{|c|c|c|c|c|c|c|c|c|c|}
\hline \multirow[t]{2}{*}{ Taxons } & \multicolumn{3}{|c|}{ Chevrens $(n=44)$} & \multicolumn{3}{|c|}{ Tougues $(n=55)$} & \multicolumn{3}{|c|}{ Nyon $(n=60)$} \\
\hline & 1994 & 1999 & $\mathrm{P}\left(\mathrm{Chi}^{2}\right)$ & 1994 & 1999 & $\mathrm{P}\left(\mathrm{Chi}^{2}\right)$ & 1994 & 1999 & $\mathbf{P}\left(\mathrm{Chi}^{2}\right)$ \\
\hline Bichaeta sanguinea & 2.3 & 0 & 1.00 & 21.8 & 12.7 & 0.207 & 21.7 & 40.0 & 0.029 \\
\hline Stylodrilus heringianus & 27.3 & 27.3 & 1.00 & 36.4 & 30.9 & 0.544 & 33.3 & 26.7 & 0.425 \\
\hline Stylodrilus lemani & 0 & 0 & - & 0 & 3.6 & 0.495 & 15.0 & 8.3 & 0.255 \\
\hline Spirosperma velutinus & 2.3 & 11.4 & 0.101 & 5.5 & 20.0 & 0.022 & 31.7 & 28.3 & 0.690 \\
\hline Potamothrix vejdovskyi & 95.5 & 84.1 & 0.159 & 90.9 & 81.8 & 0.164 & 81.7 & 55.0 & 0.001 \\
\hline Psammoryctides barbatus & 6.8 & 9.1 & 0.500 & 18.2 & 9.1 & 0.164 & 10.0 & 10.0 & 1.000 \\
\hline Spirosperma ferox & 0 & 2.3 & 0.311 & 7.3 & 5.5 & 0.696 & 5.0 & 16.7 & 0.039 \\
\hline Limnodrilus hoffmeisteri & 15.9 & 22.7 & 0.418 & 25.5 & 40.0 & 0.100 & 20.0 & 41.7 & 0.010 \\
\hline $\begin{array}{l}\text { Limnodrilus profundicola } \\
\text { Potamothrix moldaviensis }\end{array}$ & & & & & & & & & \\
\hline $\begin{array}{l}\text { Potamothrix heuscheri } \\
\text { Potamothrix hammoniensis }\end{array}$ & 93.2 & 88.6 & 0.458 & 85.5 & 80.0 & 0.499 & 73.3 & 81.7 & 0.274 \\
\hline Tubifex tubifex & & & & & & & & & \\
\hline Oligochètes oligotrophes & 29.5 & 31.8 & 0.817 & 50.9 & 47.3 & 0.703 & 70.0 & 70.0 & 1.000 \\
\hline Chironomidés & 45.5 & 54.5 & 0.394 & 43.6 & 72.7 & 0.001 & 31.7 & 68.3 & 0.001 \\
\hline Micropsectra & - & 15.9 & - & - & 47.3 & - & - & 45.0 & - \\
\hline Procladius & - & 31.8 & - & - & 36.4 & - & - & 23.3 & - \\
\hline
\end{tabular}

L'épaisseur moyenne par transect de la couche de sédiment noir qui est utilisée pour estimer la sédimentation organique, diminue d'ailleurs significativement tant en $1994\left(r_{s}=-0.80\right)$ qu'en $1999\left(r_{s}=-0.77\right)$ entre les transects 1 et 22 (Fig. 1), soit entre la limite sud de Chevrens et la limite nord de Nyon. L'abondance relative moyenne par transect des espèces d'oligochètes indicatrices de conditions oligotrophes présente la tendance opposée $\left(r_{s}=0.85\right.$ en 1994, $r_{s}=0.74$ en 1999). La figure 2 illustre la relation inverse entre ces deux variables, confirmant le lien qui existe entre la sédimentation organique et la composition des communautés d'oligochètes (Lang 1998). Cette figure permet également de classer les 22 transects et les trois bassins les uns par rapport aux autres.

Dans les 159 stations, l'abondance relative moyenne des espèces d'oligochètes indicatrices de conditions oligotrophes, calculée carotte par carotte (Tableau 3), passe de $16.8 \%$ en 1994 à $20.0 \%$ en 1999 , mais cette différence n'est pas significative (test de $t$, stations appariées, $\mathrm{P}=0.131$ ). De même, lorsque les résultats des deux campagnes 1994 et 1999 sont comparés bassin par bassin, même si les abondances augmentent, les différences ne sont pas significatives. Dans le bassin de Nyon, l'abondance relative des espèces d'oligochètes oligotrophes observée (Tableau 3) est proche de la valeur calculée à partir des concentrations en phosphore total ; c'est dans le bassin de Chevrens que la dif-



Fig. 2. Relation entre l'abondance relative (\%) moyenne des espèces d'oligochètes indicatrices de conditions oligotrophes et l'épaisseur $(\mathrm{cm})$ moyenne de la couche de sédiment noir dans les 22 transects visités en 1994 et 1999 (résultats 1994 et 1999 cumulés par transect). Les 22 transects sont identifiés par leurs numéros (voir Fig. 1). Bassin de Chevrens : transects 1 à 7, bassin de Tougues : transects 8 à 15, bassin de Nyon : transects 16 a 22. La courbe correspond à une régression robuste (LOWESS).

Fig. 2. Relationship between mean relative abundance (\%) of oligochaete species indicative of oligotrophic conditions and the mean thickness $(\mathrm{cm})$ of the black sedimentary layer in the 22 transects sampled in 1994 and in 1999 (results for both years pooled by transect). The 22 transects are identified by numbers ( see Fig. 1). Chevrens basin : transects 1 to 7, Tougues basin : transects 8 to 15 , Nyon basin : transects 16 to 22 . The curve corresponds to a robust regression (LOWESS). 
Tableau 3. Evolution de l'abondance relative moyenne (\%) des espèces d'oligochètes indicatrices de conditions oligotrophes dans le Léman entre 1978 et 1999. Sources : Lang (1998, 1999 b), cette étude.

Table 3. Changes in the mean relative abundance $(\%)$ of oligochaete species indicative of oligotrophic conditions in Lake Geneva between 1978 and 1999. Sources : Lang $(1998,1999$ b), this study.

\begin{tabular}{|c|c|c|c|c|c|c|c|}
\hline \multirow[b]{3}{*}{ Année } & \multirow[b]{3}{*}{ EOC1) } & \multicolumn{5}{|c|}{ Abondance (\%) des espèces indicatrices } & \multirow[b]{3}{*}{ Nyon } \\
\hline & & \multicolumn{2}{|c|}{$\because$ Grand Lac } & \multicolumn{3}{|c|}{ Petit Lac } & \\
\hline & & $40 \mathrm{~m}$ & $150 \mathrm{~m}$ & Total & Chevrens & Tougues & \\
\hline 1978 & 12.4 & & & 0.5 & & & \\
\hline 1982 & 8.8 & 16.9 & & & & & \\
\hline 1983 & 8.6 & & 9.5 & & & & \\
\hline 1985 & 9.9 & & & 7.9 & 1.7 & 7.4 & 13.9 \\
\hline 1988 & 12.6 & & 21.4 & & & & \\
\hline 1989 & 13.9 & & & 3.4 & 1.0 & 3.5 & 4.9 \\
\hline 1990 & 16.4 & & 24.5 & & & & \\
\hline 1991 & 19.3 & 41.3 & & & & & \\
\hline 1993 & 25.8 & & 18.7 & & & & \\
\hline 1994. & 28.2 & & & 16.8 & 4.2 & 12.8 & 29.7 \\
\hline 1996 & 32.2 & 44.6 & & & & & \\
\hline 1998 & 35.2 & & 29.9 & & & & \\
\hline 1999 & 36.6 & & & 20.0 & 8.4 & 16.7 & 31.6 \\
\hline $1999^{2}$ & 36.6 & & & 24.7 & 10.9 & 24.1 & 35.5 \\
\hline
\end{tabular}

1. Abondance des espèces oligotrophes calculee (EOC) à partir des concentrations en phosphore dans l'eau (voir Stations et méthodes).

2. Valeurs pour le Petit Lac basées sur l'abondance combinée des espèces d'oligochètes et de Chironomidés indicatrices de conditions oligotrophes.

férence est la plus grande. Lorsque l'abondance des oligochètes et des Chironomidés indicateurs de conditions oligotrophes est combinée, la valeur observée en 1999 dans le bassin de Nyon (35.5\%) est très proche de la valeur calculée $(36.6 \%)$.

\section{Discussion}

La diminution de l'abondance des oligochètes et l'augmentation de celle des larves de Chironomidés montrent clairement (Wiederholm 1980) une amélioration des sédiments profonds du Petit Lac entre 1994 et 1999. L'abondance relative moyenne des espèces d'oligochètes indicatrices de conditions oligotrophes n'augmente toutefois pas de façon significative (Tableau 3). Pour observer une augmentation significative, il faut combiner l'abondance relative des oligochètes et des larves de Chironomidés (Tableau 1). Une situation analogue a été observée dans le lac de Neuchâtel à $-40 \mathrm{~m}$ de profondeur (Lang 1999 a) : l'abondance des larves de Chironomidés indicatrices de conditions oligotrophes, en particulier celle de $\mathrm{Mi}$ cropsectra, a augmenté fortement entre 1992 et 1997 tandis que celle des oligochètes oligotrophes a diminué. Cette réaction contrastée a été attribuée à l'impact d'algues planctoniques de grande taille (surtout des diatomées) qui se sont déposées intactes à la surface des sédiments profonds (Lang 1999a). Ces algues auraient avantagé les Chironomidés en leur fournissant une nourriture abondante et désavantagé les espèces d'oligochètes adaptées à des conditions oligotrophes en réduisant les concentrations en oxygène à l'intérieur du sédiment où se développent leurs œufs. De plus, les Chironomidés adultes sont aériens et pondent leurs oeufs à la surface de l'eau ce qui leur permet de recoloniser plus rapidement le fond d'un lac que les oligochètes qui se reproduisent à l'intérieur du sédiment sans le quitter (Brinkhurst 1974). La rapide recolonisation des bassins de Tougues et de Nyon par les Chironomidés entre 1994 et 1999 confirme ce fait (Tableau 2). Pour compléter la comparaison, le phytoplancton du Léman avant la campagne de 1999, comme celui du lac de Neuchâtel avant celle de 1997, se caractérise également par la présence d'algues de grande taille (soit des algues filamenteuses : Mougeotia en 1996, Tribonema en 1997, soit des colonies d'Aphanothece en 1998) qui se sont déposées en masse sur les sédiments profonds (Druart et al. 1999). Ces algues pourraient avoir produit les mêmes effets sur le zoobenthos dans les deux lacs.

Les abondances relatives moyennes des espèces d'oligochètes oligotrophes observées en 1999 (Tableau 
3), comparées avec les valeurs de référence données dans la section Stations et méthodes, indiquent que les conditions sont celles d'un lac mésotrophe dans le bassin de Nyon et d'un lac méso-eutrophe dans le bassin de Chevrens, le bassin de Tougues se situant entre les deux. La situation observée en 1994 est comparable, mais avec des valeurs moyennes plus basses. Les prélèvements effectués dans le Petit Lac avant 1994 (Tableau 3), ne couvrant qu'une partie de la zone profonde, ne sont pas directement comparables. Toutefois, la faible abondance des espèces indicatrices suggère qu'en 1978 et, dans une moindre mesure, en 1985 et 1989 les conditions étaient beaucoup plus défavorables qu'en 1994 ou 1999 et se rapprochaient de celles d'un lac eutrophe. Cette tendance suit tout à fait l'évolution des concentrations en phosphore total dans l'eau du Léman qui, en augmentation entre 1957 et 1979 , ont diminué de façon continue entre 1979 et 1998 (Blanc et al. 1999).

En 1999, l'abondance moyenne des espèces d'oligochètes oligotrophes dans l'ensemble du Petit Lac est plus basse que les valeurs observées dans le Grand Lac à $-40 \mathrm{~m}$ et à $-150 \mathrm{~m}$ de profondeur (Tableau 3 ). La valeur observée dans la fosse de Nyon est proche de celle obtenue en 1998 à -150 m dans le Grand Lac. La comparaison des abondances observées et calculées (EOC : Tableau 3) indique que les communautés d'oligochètes du Grand Lac ont été moins affectées par l'augmentation des concentrations en phosphore que celles du Petit Lac et ont réagi plus rapidement à la diminution de cet élément. Ces réactions contrastées du zoobenthos confirment que la sédimentation organique n'est pas semblable dans les zones étudiées (Lang 1998). Comme l'accumulation de la matière organique dépend de la pente du fond (Blais \& Kalff 1995), les prélèvements du Grand Lac sont effectués dans des zones $(-40 \mathrm{~m}$ et $-150 \mathrm{~m})$ où la matière organique peut s'écouler et se déposer (focusing) au delà de $172 \mathrm{~m}$, profondeur moyenne de ce bassin (Lang 1998). Par contre dans le Petit Lac, la majorité des prélèvements sont localisés en-dessous de la profondeur moyenne $(-41 \mathrm{~m})$, soit dans une zone d'accumulation. Cela peut expliquer la lenteur de la restauration biologique des sédiments.

Dans le Petit Lac, l'influence de la sédimentation organique sur la composition du zoobenthos est manifeste tant au niveau des transects (Fig. 2) qu'au niveau des trois bassins (Tableau 2). La sédimentation organique diminuant du bassin de Chevrens à celui de Nyon, la façon dont la fréquence des taxons change d'un bassin à l'autre permet d'évaluer la valeur indicatrice du zoo- benthos. Stylodrilus heringianus confirme sa position d'espèce oligotrophe relativement résistante, par sa fréquence semblable dans les trois bassins, et Spirosperma velutinus celle de meilleur indicateur de conditions oligotrophes, par sa fréquence en augmentation entre les bassins de Chevrens et de Nyon. L'évolution de Potamothrix vejdovskyi dans le bassin de Nyon en 1999 montre que, comme dans le Grand Lac en 1996 (Lang 1998), c'est la diminution de la fréquence de cette espèce mésotrophe qui indique une amélioration de l'état des sédiments. L'augmentation significative de la fréquence de Limnodrilus hoffmeisteri et de L. profundicola entre 1994 et 1999 dans le bassin de Nyon est plus difficile à expliquer, puisqu'il s'agit d'espèces plutôt indicatrices de conditions eutrophes. Une première interprétation possible serait d'attribuer l'augmentation de la fréquence, non pas à Limnodrilus, mais à Potamothrix moldaviensis dont les immatures sont très semblables à ceux de Limnodrilus. Comme cette espèce mésotrophe est en train de coloniser le Léman (Lang 1998), l'extension de son aire de répartition dans la zone profonde n'est guère étonnante. Une autre interprétation consisterait à remettre en question la valeur indicatrice de ces deux espèces de Limnodrilus, tout au moins dans la zone profonde des lacs. En effet, dans le lac eutrophe de Morat (Lang \& Reymond 1993), ces espèces présentes jusqu'à $-20 \mathrm{~m}$ sont $\mathrm{ab}$ sentes de la zone la plus profonde (-40 m à $-45 \mathrm{~m})$ qui est colonisée seulement par Tubifex tubifex et Potamothrix hammoniensis. Les espèces du genre Limnodrilus, colonisant le Léman au delà de $-150 \mathrm{~m}$ (Lang 1998), ce n'est pas la profondeur qui limite leur distribution verticale dans le lac de Morat, mais plutôt le manque d'oxygène. Si cette interprétation est correcte, l'évolution observée dans le Petit Lac en 1999 signifierait donc que les conditions d'oxygénation se sont améliorées au niveau du fond. A ce propos, il faut remarquer que, s'il y a toujours plus de $8 \mathrm{mg} / \mathrm{l}$ d'oxygène dans l'eau du Petit Lac (Blanc et al. 1996), cette concentration, mesurée à $+1 \mathrm{~m}$ du fond, ne correspond souvent pas à celle de l'interface eau-sédiment où la consommation d'oxygène par le sédiment provoque parfois la formation d'une mince couche $(5-10 \mathrm{~mm})$, pratiquement désoxygénée ; ce qui entrave ou perturbe le développement des organismes caractéristiques des lacs oligotrophes (Müller 1992).

Dans le Petit Lac, comme dans le lac de Neuchâtel (Lang 1999 a), l'utilisation simultanée des Chironomidés et des oligochètes permet d'apprécier l'évolution des sédiments profonds mieux que ne le ferait un seul de ces groupes d'organismes. C'est d'ailleurs l'indicateur le plus simple du point de vue niveau d'identification requis, à savoir la baisse de l'abondance globale 
des oligochètes et l'augmentation de celle des Chironomidés, qui montre le mieux l'amélioration de l'état des sédiments du Petit Lac entre 1994 et 1999. Les oligochètes reflétant les conditions qui régnent à l'intérieur du sédiment et les Chironomidés celles à sa surface, leur utilisation simultanée permet de comprendre à la fois l'évolution du lac à long et à court terme. La composition du zoobenthos indique qu'en 1999 les sédiments d'une partie de la zone profonde du Petit Lac se trouvent à mi-chemin entre l'état méso-eutrophe du début des années 80 et l'état oligotrophe des années 40 . L'augmentation du pourcentage des espèces indicatrices de conditions oligotrophes dans le zoobenthos montre que la quantité et/ou la qualité de la matière organique qui se dépose à la surface du sédiment ont diminué même si la baisse des concentrations en phosphore dans l'eau n'a pas entraîné directement celle de la biomasse et de la production du phytoplancton (Druart et al. 1999, Pelletier \& Leboulanger 1999). Le zoobenthos montre clairement que le Léman est en voie de restauration parce qu'il intègre mieux que le phytoplancton les variations à long terme du milieu.

\section{Remerciements}

L'aide d'Olivier Reymond en laboratoire et celle de Laurent Cavallini sur le terrain m'ont permis de mener à bien ce travail. Les remarques du comité de lecture ont facilité la révision de cet article.

\section{Travaux cités}

Baines S.T. \& Pace M. 1994. - Relationships between suspended particulate matter and sinking flux along a trophic gradient and implication for the fate of planktonic primary production. Can.J. Fish. Aquat. Sci., $51: 25-36$.

Blais J.M. \& Kalff J. 1995. - The influence of lake morphometry on sediment focusing. Limnol. Oceanogr., $40: 582-588$.

Blanc P., Corvi C., Nirel P., Revaclier R. \& Rapin F. 1996. - Evolution physico-chimique des eaux du Léman, campagne 1995. Rapport de la commission internationale pour la protection des eaux du Léman contre la pollution : 37-80.

Blanc P., Corvi C., Khim-Heang S. \& Rapin F. 1999. - Evolution chimique des eaux du Léman. Rapport de la commission internationale pour la protection des eaux du Léman contre la pollution : 33-59.

Brinkhurst R.O. 1974. - The benthos of lakes. The Macmillan Press, London Basingtoke : $190 \mathrm{p}$.

Demierre A. \& Durand P. 1999. - La végétation macrophytique du Léman. Rapport de la commission internationale pour la protection des eaux du Léman contre la pollution : 129-217.

Druart J.-C., Revaclier R., Pelletier J.P. \& Vansteelant-el Jay A. 1999. - Evolution du phytoplancton du Léman. Rapport de la commission internationale pour la protection des eaux du Léman contre la pollution : 69-78.

Hakanson L. \& Jansson M. 1983. - Principles of lake sedimentolo- gy. Springer Verlag, Berlin/Heidelberg/New York/Tokio : $316 \mathrm{p}$. Johnson R. K., Wiederholm T. \& Rosenberg D. M. 1993. - Freshwater biomonitoring using individual organisms, populations, and species assemblages of benthic macroinvertebrates. Rosenberg, D.M. \& Resh V.H. (eds). in Freshwater biomonitoring and benthic macroinvertebrates. Chapman \& Hall. New York : 488 p.

Juget J. 1967. — La faune benthique du Léman : modalités et déterminisme écologiques du peuplement. Thèse univ. Lyon : $366 \mathrm{p}$.

Lang C. 1990. - Quantitative relationships between oligochaete communities and phosphorus concentrations in lakes. Freshwat. Biol., 24 : 327-334.

Lang C. 1998. - Using oligochaetes to monitor the decrease of eutrophication : the 1982 - 1996 trend in Lake Geneva. Arch Hydrobiol., $141:$ :447-458.

Lang C. 1999a. - Contrasting responses of oligochaetes (Annelida) and Chironomids (Diptera) to the abatement of eutrophication in Lake Neuchâtel. Aquatic Sciences, 61 : 206-214.

Lang C. 1999b. - Evolution de l'état trophique du Léman entre 1990 et 1998 indiquée par les communautés de vers présentes à $150 \mathrm{~m}$ de profondeur. Rapport de la commission internationale pour la protection des eaux du Léman contre la pollution : 101-110.

Lang C. \& Reymond O. 1993. - Eutrophisation du lac de Morat indiquée par les communautés d'oligochètes : tendance 1980 - 1991. Rev. suisse Zool., 100 (1) : 11-18.

Lang C. \& Reymond O. 1996a. - Empirical relationships between oligochaetes, phosphorus and organic deposition during the recovery of Lake Geneva from eutrophication. Arch. Hydrobiol., 136 :237-245.

Lang C. \& Reymond O. 1996b. - Etat trophique de la région ouest du Léman indiqué par les communautés de vers : résultats 1994 et 1995. Rapport de la commission internationale pour la protection des eaux du Léman contre la pollution : 167-172.

Lemmin U., Perrinjaquet C. \& Graf W.H. 1989. - Etude de la variation saisonnière des phénomènes de mélange dans l'hypolimnion du Léman. Rapport de la commission internationale pour la protection des eaux du Léman contre la pollution : 89-105.

Lods-Crozet B. \& Lachavanne J.-B. 1994. - Changes in the chironomid communities in Lake Geneva in relation with eutrophication, over a period of 60 years. Arch. Hydrobiol., $130: 453-471$.

Müller R. 1992. - Trophic state and its implications for natural reproduction of salmonid fish. Hydrobiologia, 243/244 : 261-268.

Pelletier J. \& Leboulanger Ch. 1999. - Evolution de la production phytoplanctonique dans le Léman. Rapport de la commission internationale pour la protection des eaux du Léman contre la pollution : 61-68.

Reymond O. 1994. - Préparations microseopiques permanentes d'oligochètes : une méthode simple. Bull. Soc. Vaud. Sc. Nat., 83 : 1-3.

Saether O.A. 1979. - Chironomid communities as water quality indicators. Holarct. Ecol., 2 : 65-74.

Sas H. 1989. - Lake restoration by reduction of nutrient loading. Academia Verlag Richarz GmbH. St. Augustin : 497 p.

Wiederholm T. 1980. - Use of benthos in lake monitoring. $J$. WPCF 52 : 537-547.

Wiederholm T. (Ed.) 1983. - Chironomidae of the Holarctic region. Part 1. Larvae. Entomologica Scandinavica. Suppl. $19: 457$ p. 